\title{
Increased Risk of Osteoporosis in Gastric Cancer Survivors Compared to General Population Control: A Study with Representative Korean Population
}

\author{
Su-Min Jeong, $\mathrm{MD}^{1}$ \\ Dong Wook Shin, MD, DrPH, MBA² \\ Ji Eun Lee, MD, MPH ${ }^{1}$ \\ Sang-Man Jin, MD, $P h D^{3}$ \\ Sung Kim, MD, PhD ${ }^{4}$
}

${ }^{1}$ Department of Family Medicine, Seoul National University Hospital, Seoul, ${ }^{2}$ Department of Family Medicine and Supportive Care Center, Samsung Medical Center, Seoul, ${ }^{3}$ Division of Endocrinology and Metabolism and ${ }^{4}$ Department of Surgery, Samsung Medical Center, Sungkyunkwan University School of Medicine, Seoul, Korea

\section{Purpose}

Although several studies have suggested that osteoporosis is common in survivors of gastric cancer (GC), no study to date has directly assessed the risk for osteoporosis in GC survivors compared to matched controls. Thus, we aimed to investigate the relative risk for osteoporosis in survivors of GC compared to general population.

\section{Materials and Methods}

We used the Korea National Health and Nutrition Examination Survey data (2008-2011). Patients with a history of $\mathrm{GC}(\mathrm{n}=94)$ were defined as case among 8,142 individuals over 50 years old who were evaluated by dual-energy $X$-ray absorptiometry. Controls $(n=470)$ were matched to cases by age and sex in a 1:5 ratio. Osteopenia $(-2.5<$ T-score $<-1.0)$ and osteoporosis (T-score $\leq-2.5$ ) were defined.

\section{Results}

The prevalence of osteoporosis in GC survivors was $30.2 \%$, which was significantly greater than that of controls (19.7\%). In total, GC survivors had a 3.7-fold increased risk for osteoporosis compared to controls $(p=0.021)$. In addition, the risk for osteoporosis of the total proximal femur total (TF) and femur neck (FN) was significantly increased among GC survivors compared to controls (adjusted relative risk, 4.64; 95\% confidence interval, 1.16 to 18.6 in TF and adjusted relative risk, 3.58; 95\% confidence interval, 1.19 to 10.8 in FN). Furthermore, we found sub-optimal daily calcium intake and mean serum levels of 25hydroxy-vitamin $\mathrm{D}$ in both groups.

\section{Conclusion}

GC survivors are at significantly increased risk for osteoporosis, especially in the femur. Clinically, our finding supports the importance of screening bone health and adequate nutrient supplementation in survivors of GC.
Correspondence: Dong Wook Shin, MD, DrPH, MBA Department of Family Medicine, Samsung Medical Center, Supportive Care Center, Samsung Comprehensive Cancer Hospital, 81 Irwon-ro, Gangnam-gu, Seoul, Korea Tel: 82-2-6190-5252

Fax: 82-2-3410-2459

E-mail: dwshin.md@gmail.com

Co-correspondence: Ji Eun Lee, MD, MPH Department of Family Medicine,

Seoul National University Hospital, 101 Daehak-ro, Jongno-gu, Seoul 03080, Korea Tel: 82-2-8883-9984

Fax: 82-2-766-3296

E-mail: jieun10@gmail.com

Received March 19, 2018

Accepted June 25, 2018

Published Online June 27, 2018
Key words

Osteoporosis, Gastric neoplasms, Calcium, Vitamin D 


\section{Introduction}

As of 2012, gastric cancer was the fifth most common cancer worldwide [1]. Although the incidence of gastric cancer has decreased rapidly in Western countries, it remains the second most common cancer in Asia [2]. In South Korea, gastric cancer is the second leading cause of cancer behind thyroid cancer [3]. However, owing to increasing rates of participation in national gastric cancer screening programs [4] and development of endoscopic examinations leading to earlier detection and initiation of treatment [5], more than half of gastric cancers detected in Korea are identified at T1 stage without lymph node metastasis. Advancements in treatment modalities for gastric cancer including extensive lymph node dissection and availability of effective chemotherapy regimens has led to an increase in long-term survival [6]. Indeed, the 5-year survival rate for all gastric cancer is now $67 \%$, while that of early stage gastric cancer is $92 \%$ [7]. This improvement in survival rates for gastric cancers has heralded significant attention as a model of how to address and appropriately mange long-term health problems.

The sequelae of undergoing treatment for gastric cancer include malnutrition, poor bone health and decreased quality of life [8-10]. Poor bone health predisposes patients to osteoporosis and an increased risk of fracture, which can cause physical disability and is associated with a high rate of mortality [11]. Indeed, several previous studies have reported a high prevalence of osteoporosis $[9,12]$ and osteoporotic fracture $[13,14]$ in survivors of gastric cancer post-gastrectomy. Lim et al. [9] reported that the prevalence of osteoporosis after gastrectomy was $38.3 \%$ among 133 gastric cancer survivors and compared factors that predict osteoporosis stratified by sex among gastric cancer survivors, but not with healthy controls. Similarly, Yoo et al. [12] investigated the prevalence of osteoporosis and associated factors in gastric cancer survivors. In their study, they indirectly compared the prevalence of osteoporosis in gastric cancer survivors with that of the general population based on the results of other studies but did not consider nutrient intake or nutrient status. Lastly, in the Mediterranean Osteoporosis Study, the relative risk for hip fracture in men who underwent gastrectomy due to all gastric diseases including cancer was 1.78 (95\% confidence interval [CI], 1.13 to 2.85 ) compared to controls [14].

Studies directly comparing bone health between patients with gastric cancer and that of the general population are rare. Therefore, this aim of this study was to assess the relative risk for osteoporosis in gastric cancer survivors compared to the general population free of cancer using data from the Korea National Health and Nutrition Examination Survey (KNHNES).

\section{Materials and Methods}

\section{Study population}

We used KNHNES data collected between July 2008 and May 2011. KNHNES is a nationwide representative crosssectional survey consisting of a clustered, multistage, stratified, probability-sampling design using household registries. The survey includes demographic, socioeconomic, medical, and dietary information [15]. The survey questionnaires were administrated by trained investigators except information related to health behaviors that was performed based on selfreported questionnaires.

Among an initial dataset comprising 21,303 subjects, we selected 8,142 individuals who had undergone dual-energy X-ray absorptiometry (DXA) and were more than 50 years old. We excluded 330 individuals with a history of cancer other than gastric cancer, leaving 7,812 subjects, from which a total of 94 gastric cancer survivors were identified based on the question: "Have you ever been diagnosed with gastric cancer by a doctor?" Four hundred seventy controls who were free of any cancers were matched by 1:5 ratio for each case of gastric cancer with exact age and sex. In subgroup analysis, gastric cancer survivors were divided into two groups according to the period after gastric cancer diagnosis ( $\leq 5$ years, $>5$ years).

\section{Diagnosis of osteoporosis and osteopenia}

Bone mineral density (BMD) at the total proximal femur (TF), femoral neck (FN), and lumbar spine (LS; first to fourth lumbar spine) was measured by DXA (Hologic Inc., Bedford, MA). T-scores were calculated among postmenopausal women and men more than 50 years or 50 years old using the Asian (Japanese) reference curve supplied by the manufacturer. Osteoporosis and osteopenia were defined by T-score according to the World Health Organization definition [16]. A normal density was defined as T-score $\geq-1.0$, osteopenia was defined as a T-score between -1.0 and -2.5 , and osteoporosis was defined as T-score $\leq-2.5$.

\section{Additional variables}

Body weight and height were measured to the nearest 0.1 $\mathrm{kg}$ and $0.1 \mathrm{~cm}$, respectively, with subjects wearing light clothing without shoes. Body mass index (BMI) was calculated as weight in kilograms divided by height in meters squared and was analyzed as a continuous variable. Smoking status was categorized as never smoker, former, and current smoker based on self-reported questionnaire. Alcohol consumption was classified by frequency as $<2$ times per week or $\geq 2$ times 
Table 1. Baseline characteristics of the study population

\begin{tabular}{|c|c|c|c|}
\hline Variable & $\begin{array}{l}\text { No gastric cancer } \\
\qquad(\mathrm{n}=470)\end{array}$ & $\begin{array}{c}\text { Gastric cancer } \\
\text { survivor }(n=94)\end{array}$ & p-value \\
\hline Age (yr) & $65.7 \pm 0.5$ & $66.5 \pm 1.1$ & 1.00 \\
\hline \multicolumn{4}{|l|}{ Sex } \\
\hline Male & $330(70.4)$ & $66(68.8)$ & 1.00 \\
\hline Female & $140(29.6)$ & $28(31.2)$ & \\
\hline Height $(\mathrm{cm})$ & $162.1 \pm 0.5$ & $161.3 \pm 1.3$ & 0.916 \\
\hline Weight (kg) & $63.0 \pm 0.6$ & $58.5 \pm 1.3$ & $<0.001$ \\
\hline Body mass index $\left(\mathrm{kg} / \mathrm{m}^{2}\right)$ & $23.9 \pm 0.2$ & $22.4 \pm 0.5$ & $<0.001$ \\
\hline \multicolumn{4}{|l|}{ Household income ${ }^{a)}$} \\
\hline Highest quartile & $70(19.4)$ & 11 (11.6) & 0.818 \\
\hline Third qurtile & $90(18.3)$ & $16(18.0)$ & \\
\hline Second quartile & $138(29.6)$ & $27(35.2)$ & \\
\hline Lowest quartile & $170(32.7)$ & $37(35.2)$ & \\
\hline \multicolumn{4}{|l|}{ Smoking status } \\
\hline Never smoker & $180(38.6)$ & $32(33.4)$ & 0.437 \\
\hline Former and current smoker & $290(61.4)$ & $62(66.6)$ & \\
\hline \multicolumn{4}{|l|}{ Alcohol consumption (per week) } \\
\hline$<2$ & $331(70.7)$ & $76(77.0)$ & 0.040 \\
\hline$\geq 2$ & $139(29.3)$ & $18(23.0)$ & \\
\hline \multicolumn{4}{|l|}{ Moderate to intense physical activity } \\
\hline Yes & $350(72.7)$ & $74(75.4)$ & 0.383 \\
\hline No & $120(27.3)$ & $20(24.6)$ & \\
\hline \multicolumn{4}{|l|}{ Hypertension } \\
\hline No & $228(49.9)$ & $56(50.1)$ & 0.050 \\
\hline Yes & $242(62.3)$ & $38(37.7)$ & \\
\hline \multicolumn{4}{|l|}{ Diabetes } \\
\hline No & $368(78.3)$ & $78(85.8)$ & 0.308 \\
\hline Yes & $102(21.7)$ & $16(14.2)$ & \\
\hline \multicolumn{4}{|c|}{ Undergoing health check-ups for recent 2 years } \\
\hline Yes & $296(63.1)$ & $67(69.4)$ & 0.125 \\
\hline No & $174(36.9)$ & $27(30.6)$ & \\
\hline Calcium intake (mg/day) $\left.{ }^{b}\right)$ & $481.0 \pm 20.8$ & $439.2 \pm 39.7$ & 0.424 \\
\hline$\geq 800$ & $60(13.0)$ & $17(11.9)$ & 0.798 \\
\hline$<800$ & $384(87.0)$ & $77(88.1)$ & \\
\hline 25-Hydroxy-vitamin D (ng/mL) & $20.2 \pm 0.4$ & $17.9(1.2)$ & 0.022 \\
\hline$\geq 20$ & $206(45.7)$ & $33 \pm 37.6$ & 0.170 \\
\hline$<20$ & $233(54.3)$ & $52(62.4)$ & \\
\hline Alkaline phosphatase (IU/L) ${ }^{c}$ & $246.6 \pm 6.1$ & $279.7 \pm 11.5$ & 0.003 \\
\hline$<250$ & $282(63.9)$ & $36(42.6)$ & $<0.001$ \\
\hline$\geq 250$ & $157(36.1)$ & $49(57.4)$ & \\
\hline Parathyroid hormone $(\mathrm{pg} / \mathrm{mL})^{\mathrm{d})}$ & $68.7 \pm 1.7$ & $73.7 \pm 5.8$ & 0.198 \\
\hline$<65$ & $237(54.4)$ & $41(47.6)$ & 0.384 \\
\hline$\geq 65$ & $202(45.6)$ & $43(52.4)$ & \\
\hline
\end{tabular}

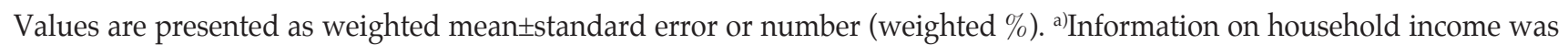
obtained in 559 subjects (468 controls and 91 cases), b)Information on calcium intake was obtained in 532 subjects (444 controls and 88 cases), c)Information on vitamin D levels and alkaline phosphatase and parathyroid hormone were obtained in 524 subjects (439 controls and 84 cases), d)Information on parathyroid hormone was obtained in 523 subjects (439 controls and 83 cases). 
Table 2. Comparison of bone mineral density and T-scores between gastric cancer survivors and controls

\begin{tabular}{|c|c|c|c|}
\hline & $\begin{array}{l}\text { No gastric cancer } \\
\qquad(n=470)\end{array}$ & $\begin{array}{l}\text { Gastric cancer } \\
\text { survivor }(n=94)\end{array}$ & p-value \\
\hline \multicolumn{4}{|l|}{ Bone mineral density $\left(\mathrm{g} / \mathrm{cm}^{2}\right)$} \\
\hline Total femur & $0.87 \pm 0.01$ & $0.80 \pm 0.02$ & $<0.001$ \\
\hline Femur neck & $0.70 \pm 0.01$ & $0.65 \pm 0.02$ & 0.003 \\
\hline Lumbar spine & $0.90 \pm 0.01$ & $0.83 \pm 0.02$ & $<0.001$ \\
\hline \multicolumn{4}{|l|}{ T-score } \\
\hline Normal $(\geq-1.0)$ & $150(34.4)$ & $15(14.4)$ & 0.002 \\
\hline Osteopenia $(-2.5$ to -1.0$)$ & $224(45.9)$ & $48(55.4)$ & \\
\hline Osteoporosis $(\leq-2.5)$ & $96(19.7)$ & $31(30.2)$ & \\
\hline
\end{tabular}

Values are presented as weighted mean \pm standard error or number (weighted \%).

per week. Physical activity was classified based on whether subjects engaged in intense to moderate physical activity for at least 10 minutes during the prior week or not. Hypertension was defined as those who were taking anti-hypertensive drug, had hypertension diagnosis history or systolic blood pressure $\geq 140 \mathrm{~mm} \mathrm{Hg}$, or diastolic blood pressure $\geq 90 \mathrm{~mm}$ $\mathrm{Hg}$. Diabetes was defined as taking hypoglycemic agents, having fasting blood glucose $\geq 126 \mathrm{mg} / \mathrm{dL}$. Quartile of household income was used to assess the socioeconomic status. Experience of health check-up within recent 2 years was asked and classified into yes or no.

In terms of nutrition intake and markers of bone metabolism, daily calcium intake was assessed using a 24-hour recall method the day before the survey and was classified by 800 $\mathrm{mg}$ according to recommended dietary allowance [17]. Fasting blood samples were used to measure serum levels of 25hydroxy-vitamin D [25(OH)D], alkaline phosphatase (ALP), and parathyroid hormone (PTH). 25(OH)D levels were categorized into two groups by $20 \mathrm{ng} / \mathrm{mL}$. ALP levels were divided by $250 \mathrm{IU} / \mathrm{L}$, and PTH levels were divided by 65 $\mathrm{pg} / \mathrm{mL}$.

\section{Statistical analysis}

To represent the entire Korean population, weights were assigned to each subject in order to give an equal probability for sampling. All statistical analyses were performed considering sample weights, which were constructed based on the survey design. All data were presented as mean with standard error or number with (weighted \%). Differences between cases and controls were analyzed using a two-tailed Student's $t$ test for continuous variables and $\chi^{2}$ test for categorical variables. Multinomial logistic regression was used to compare the relative risk for osteopenia and osteoporosis between cases and controls after adjusting for factors that could affect bone health, namely, age, sex, BMI, smoking sta- tus, alcohol consumption physical activity in model 1. Model 2 additionally adjusted hypertension, diabetes, household income and health check-up history. A multivariable logistic regression model was used to compare differences in nutritional status and biomarker levels between cases and controls and between normal and osteoporosis. All statistical analyses were performed using Stata ver. 14.1 (Stata Corp, College Station, TX).

\section{Ethical statement}

All subjects participated voluntarily and provided informed consent. The KNHANES was annually approved by the institutional review board of the Centers for Disease Control and Prevention (2008-04EXP-01-C, 2009-01CON-03-2C, 201002CON-21-C, and 2011-02CON-06-C).

\section{Results}

\section{Baseline characteristics}

The general characteristics in the controls and gastric cancer survivors are shown in Table 1 . The mean age and sex distributions were similar in both groups due to age and sex matching used to select controls. The weighted mean age was $65.7 \pm 0.5$ years in the control group and $66.5 \pm 1.1$ years in the gastric cancer survivor group. Approximately $30 \%$ of subjects were female in both groups. The mean weight of gastric cancer survivors $(58.5 \pm 1.3 \mathrm{~kg})$ was less than that of controls $(63.0 \pm 0.6 \mathrm{~kg}, \mathrm{p}<0.001)$. BMI was also lower in gastric cancer survivors compared to controls $\left(22.4 \mathrm{~kg} / \mathrm{m}^{2} \mathrm{vs}\right.$. $\left.23.9 \mathrm{~kg} / \mathrm{m}^{2}, \mathrm{p}<0.001\right)$. The prevalence of frequent alcohol consumption was higher in controls compared to gastric can- 
Table 3. Multinomial logistic regression for osteoporosis according to gastric cancer history

\begin{tabular}{|c|c|c|c|c|c|c|}
\hline & \multicolumn{2}{|c|}{ Unadjusted } & \multicolumn{2}{|l|}{ Model 1} & \multicolumn{2}{|l|}{ Model 2} \\
\hline & $\mathbf{R R}(95 \% \mathbf{C I})$ & p-value & Adjusted RR ${ }^{a}(95 \%$ CI $)$ & p-value & Adjusted $\mathbf{R R}^{\mathrm{a})}(95 \% \mathrm{CI})$ & p-value \\
\hline \multicolumn{7}{|l|}{ Overall } \\
\hline Normal & 1.00 & & 1.00 & & 1.00 & \\
\hline Osteopenia & $1.50(0.61-3.67)$ & 0.376 & $2.70(1.21-7.27)$ & 0.017 & $2.80(1.10-7.13)$ & 0.031 \\
\hline Osteoporosis & $3.30(1.26-8.66)$ & 0.015 & $3.51(1.23-10.04)$ & 0.019 & $3.72(1.22-11.4)$ & 0.021 \\
\hline \multicolumn{7}{|l|}{ Total femur } \\
\hline Normal & 1.00 & & 1.00 & & 1.00 & \\
\hline Osteopenia & $1.79(0.83-3.88)$ & 0.137 & $1.42(0.66-3.01)$ & 0.373 & $1.13(0.56-2.28)$ & 0.725 \\
\hline Osteoporosis & $8.84(2.14-36.6)$ & 0.003 & $4.30(1.21-15.3)$ & 0.024 & $4.64(1.16-18.6)$ & 0.030 \\
\hline \multicolumn{7}{|l|}{ Femur neck } \\
\hline Normal & 1.00 & & 1.00 & & 1.00 & \\
\hline Osteopenia & $1.63(0.76-3.50)$ & 0.211 & $2.11(0.98-4.57)$ & 0.057 & $1.96(0.88-4.36)$ & 0.051 \\
\hline Osteoporosis & $3.01(1.09-8.26)$ & 0.033 & $2.74(0.91-8.24)$ & 0.072 & $3.58(1.19-10.8)$ & 0.023 \\
\hline \multicolumn{7}{|l|}{ Lumbar spine } \\
\hline Normal & 1.00 & & 1.00 & & 1.00 & \\
\hline Osteopenia & $1.44(0.66-3.18)$ & 0.361 & $2.30(1.08-4.89)$ & 0.030 & $2.30(1.10-4.83)$ & 0.028 \\
\hline Osteoporosis & $2.21(0.94-5.18)$ & 0.068 & $2.27(0.93-5.55)$ & 0.073 & $2.17(0.86-5.47)$ & 0.099 \\
\hline
\end{tabular}

$\mathrm{RR}$, relative risk; CI, confidence interval. a)Model 1 was adjusted for age, sex, body mass index, smoking status, alcohol consumption, and physical activity and Model 2 was adjusted for covariates in model 1 plus hypertension, diabetes, household income and health check-up status in 559 subjects.

Table 4. Comparison of nutrient status and biomarker levels between gastric cancer survivor and controls

\begin{tabular}{|c|c|c|c|c|c|c|}
\hline & \multicolumn{2}{|c|}{ Unadjusted } & \multicolumn{2}{|c|}{ Model 1} & \multicolumn{2}{|c|}{ Model 2} \\
\hline & OR $(95 \% \mathrm{CI})$ & p-value & $\begin{array}{c}\text { Adjusted OR } \\
(95 \% \mathrm{CI})\end{array}$ & p-value & $\begin{array}{l}\text { Adjusted OR } \\
\text { (95\% CI) }\end{array}$ & p-value \\
\hline \multicolumn{7}{|c|}{ Calcium intake (mg/day) } \\
\hline$\geq 800$ & 1.00 & & 1.00 & & 1.00 & \\
\hline$<800$ & $1.11(0.50-2.43)$ & 0.801 & $1.04(0.46-2.31)$ & 0.933 & $0.80(0.36-1.81)$ & 0.598 \\
\hline \multicolumn{7}{|c|}{ 25-Hydroxy-vitamin D (ng/mL) } \\
\hline$\geq 20$ & 1.00 & & 1.00 & & 1.00 & \\
\hline$<20$ & $1.39(0.77-2.51)$ & 0.268 & $1.33(0.73-2.41)$ & 0.348 & $1.35(0.73-2.51)$ & 0.334 \\
\hline \multicolumn{7}{|c|}{ Alkaline phosphatase (IU/L) } \\
\hline$<250$ & 1.00 & & 1.00 & & 1.00 & \\
\hline$\geq 250$ & $2.39(1.32-4.30)$ & 0.004 & $2.05(1.09-3.85)$ & 0.027 & $2.30(1.19-4.44)$ & 0.013 \\
\hline \multicolumn{7}{|c|}{ Parathyroid hormone (pg/mL) } \\
\hline$<65$ & 1.00 & & 1.00 & & 1.00 & \\
\hline$\geq 65$ & $1.31(0.74-2.34)$ & 0.354 & $1.28(0.70-2.35)$ & 0.429 & $1.41(0.76-2.60)$ & 0.277 \\
\hline
\end{tabular}

OR, odds ratio; CI, confidence interval. a)Model 1 was adjusted for age, sex, body mass index, smoking status, alcohol consumption, and physical activity and Model 2 was adjusted for covariates in model 1 plus hypertension, diabetes, household income and health check-up status. 
cer survivors. There was no significant difference in the level of physical activity between controls and gastric cancer survivors.

Differences in the BMD of TF, FN, and LS are shown in Table 2. The BMDs of TF, FN, and LS were lower in the gastric cancer group compared to controls (all $\mathrm{p}<0.05$ ). Furthermore, $30.2 \%$ of gastric cancer survivors had osteoporosis, which was higher than that of the control group (19.7\%) $(\mathrm{p}=0.002)$.

\section{Risk of osteoporosis}

In total, gastric cancer survivors had a 3.7-fold increased risk for osteoporosis compared to controls ( $\mathrm{p}=0.021$ ) (Table 3 ). The risk for TF and FN osteoporosis was significantly increased in gastric cancer survivors compared to controls (adjusted relative risk [aRR], 4.64; $95 \% \mathrm{CI}, 1.16$ to $18.6 ; \mathrm{p}=0.030$ in TF and aRR, 3.58; 95\% CI, 1.19 to 10.8; $\mathrm{p}=0.023$ in FN). There was no significant difference in the risk for LS osteoporosis between the two groups $(\mathrm{p}=0.099)$.

According to the period after gastric cancer diagnosis (before or after 5 years), The risk for osteopenia and osteoporosis was significantly increased in gastric cancer survivors (aRR, 5.89; 95\% CI, 1.81 to 19.2 for osteopenia and aRR, 7.17; $95 \% \mathrm{CI}, 1.62$ to 31.8 for osteoporosis) in the group exceeding 5 years after cancer diagnosis (S1 Table).

\section{Nutrition status and biomarkers of bone metabolism}

Daily calcium intake was insufficient in more than $87 \%$ of subjects in both groups (Table 1). Only $11.9 \%$ of gastric cancer survivors satisfied the recommended level. The serum $25(\mathrm{OH}) \mathrm{D}$ level was significantly lower in gastric cancer survivors compared to controls ( $17.9 \mathrm{ng} / \mathrm{mL}$ vs. $20.2 \mathrm{ng} / \mathrm{mL}$ ) $(\mathrm{p}=0.022)$, with $37.6 \%$ of gastric cancer survivors reaching a concentration of $20 \mathrm{ng} / \mathrm{mL}$. The mean ALP level in gastric cancer survivors was higher than that of controls $(\mathrm{p}=0.003)$. Lastly, mean PTH was not significantly different between the two groups ( $\mathrm{p}=0.198)$. Gastric cancer survivors were associated with elevated ALP (adjusted odds ration [aOR], 2.30; $95 \%$ CI, 1.19 to 4.44) (Table 4). Low calcium intake and serum 25(OH)D level were not significantly associated with gastric cancer survivors compared to controls. In addition, elevated ALP and PTH levels were associated with osteoporosis compared to normal (aOR, 4.44; 95\% CI, 1.99 to 9.91) and (aOR, $2.49 ; 95 \% \mathrm{CI}, 1.15$ to 5.40$)$, respectively (S2 Table).

\section{Discussion}

To the best of our knowledge, there have been only a few studies to evaluate differences in BMD and relative risk for osteoporosis in gastric cancer survivors compared to controls. In the present study, we confirmed that BMD of the femur and lumbar spine were lower in gastric cancer survivors compared to age- and sex-matched controls, and that gastric cancer survivors have an approximately 4.6-fold and 3.6-fold increased risk for osteoporosis of TF and FN compared to controls, respectively. In addition, we found that $88.1 \%$ and $62.4 \%$ of gastric cancer survivors have inadequate levels of daily calcium intake and serum 25(OH)D levels.

Our results showed that gastric cancer survivors have a lower BMD of the TF, FN, and LS and higher prevalence $(30.2 \%)$ of osteoporosis compared to controls $(19.7 \%)$. The high prevalence of osteoporosis was consistent with previous studies $[9,12,18,19]$. A prior case-control study in 18 patients after total gastrectomy reported that the adjusted mean differences in BMD of the LS and FN were significantly less than that of healthy controls [18]. However, that study was adjusted only for height as a covariate due to relatively small sample size. In addition, Adachi et al. [19] reported that subjects who had undergone post-gastrectomy more than 5 years before had a $12.5 \%$ and $16.9 \%$ decrease of BMD of the LS in men and women compared to age- and sex-matched controls. In that study, only BMD of the LS was evaluated.

On the other hands, the prominent decrease of BMD was observed in gastric cancer survivors after 5 years from cancer diagnosis. This result suggests that osteoporosis may be a problem after gastric cancer diagnosis rather than an inherent problem before gastric cancer diagnosis.

The possible etiologies of increased risk of osteoporosis in gastric cancer survivors include low calcium intake with malabsorption, low vitamin D levels, and weight loss. Firstly, we found that calcium intake and calcium absorption were inadequate in gastric cancer survivors. Specifically, more than $88 \%$ of the patients in the present study had a daily calcium intake less than $800 \mathrm{mg}$. Consistently, a cohort study of 1,289 patients after gastrectomy reported a daily calcium intake around $600 \mathrm{mg}$ [20]. In addition, calcium absorption, which occurs primarily in the duodenum, can be interrupted due to bypassing the duodenal surface secondary to rapid transit of food [21]. Lastly, decreased gastric acidity after gastrectomy also contributes to the low absorption of calcium [22].

Low serum vitamin D level may reflect either low intake or malabsorption of vitamin $\mathrm{D}$. The reported rate of vitamin D deficiency $(<20 \mathrm{ng} / \mathrm{mL})$ in gastric cancer survivors was 61.5\% [23]. As vitamin $\mathrm{D}$ is fat-soluble, exocrine pancreatic insufficiency after gastrectomy, which causes steatorrhea, 
could aggravate vitamin D malabsorption [24].

Low calcium intake and low serum vitamin D level after gastric bypass surgery [25] or gastrectomy [22] may result in secondary hyperparathyroidism, a physiological response aimed at maintaining normal serum calcium and vitamin $\mathrm{D}$ at the expense of bone mass. As such, increased bone turnover reflected by elevated levels of PTH and ALP may reflect osteoblastic activity [26]. A hyperparathyroid response can also cause deterioration of cortical bone microstructure with a reduction in cortical bone density and cortical thickness [27], in contrast with anabolic effects on trabecular bone, which predominates at the spine [28]. Our results also demonstrated an increased risk for osteoporosis in TF and FN rather than LS.

Weight loss may increase the risk for osteoporosis in gastric cancer survivors. A previous study reported that marked weight loss after gastrectomy is associated with osteoporosis [12]. Likewise, bone loss is magnified in patients who have undergone Roux-en-Y gastric bypass, which is also known to cause the greatest degree of weight loss compared to other types of bariatric surgery [27].

Among the general population, screening rates for osteoporosis are as low as 56\% in women and 38\% in men [29]. Furthermore, $88.4 \%$ of gastric cancer survivors are unaware of the status of their bone health [30], despite the recommendations of the American Gastroenterology Association [31]. In other words, both physicians and patients appear to be generally unaware of the risks of osteoporosis in gastric cancer survivors. In terms of managing poor bone health, oral supplementation of vitamin D and calcium could be an effective method to stabilize PTH level and reduce bone loss [32].

There were several strengths of the present study, foremost of which was the use of a representative and comprehensive national database that included information on medical history, DXA, bone biomarkers, and nutrient status. The main limitation of this study was that we were not able to clarify the specific treatment history for gastric cancer, such as total gastrectomy, partial gastrectomy, or endoscopic submucosal dissection (ESD) of local early stage gastric cancers. However, patients who have undergone ESD have also been reported to have low vitamin D and calcium intake [21]. Furthermore, definition of gastric cancer survivors was based on questionnaire. Although the questionnaire was conducted by professional interviewers, the accuracy of self-reported cancer history could be low. A study reported that sensitivity of self-reported cancer history was as low as 72\% [33]. However, gastric cancer had relatively high sensitivity (78\%) and high positive predictive value (93\%). In addition, reliability for self-reported cancer history was ranged from $80 \%$ to $100 \%$ [34]. Lastly, we were not able to determine the reliability of reported histories for factors associated with bone health including fracture history, current osteoporosis treatment, and supplementation of calcium and vitamin D.

In conclusion, we found that risk for osteoporosis of TF and $\mathrm{FN}$ in gastric cancer survivors was 4.6-fold and 3.6-fold higher than that of age and sex matched controls, respectively. Overall, daily calcium intake and serum vitamin D level were deemed insufficient for maintaining bone health among study participants. Thus, gastric cancer survivors need to be screened for bone health and encouraged to maintain adequate nutrient intake.

\section{Electronic Supplementary Material}

Supplementary materials are available at Cancer Research and Treatment website (https:// www.e-crt.org).

\section{Conflicts of Interest}

Conflict of interest relevant to this article was not reported.

\section{References}

1. Ferlay J, Soerjomataram I, Dikshit R, Eser S, Mathers C, Rebelo $\mathrm{M}$, et al. Cancer incidence and mortality worldwide: sources, methods and major patterns in GLOBOCAN 2012. Int J Cancer. 2015;136:E359-86.

2. Howlader N, Noone AM, Krapcho M, Miller D, Bishop K, Altekruse SF, et al. SEER cancer statistics review, 1975-2014. Bethesda, MD: National Cancer Institute; 2016.

3. Jung KW, Won YJ, Oh CM, Kong HJ, Lee DH, Lee KH, et al. Cancer statistics in Korea: incidence, mortality, survival, and prevalence in 2014. Cancer Res Treat. 2017;49:292-305.
4. Suh M, Choi KS, Park B, Lee YY, Jun JK, Lee DH, et al. Trends in cancer screening rates among Korean men and women: results of the Korean national cancer screening survey, 20042013. Cancer Res Treat. 2016;48:1-10.

5. Jeong O, Park YK. Clinicopathological features and surgical treatment of gastric cancer in South Korea: the results of 2009 nationwide survey on surgically treated gastric cancer patients. J Gastric Cancer. 2011;11:69-77.

6. Bang YJ, Van Cutsem E, Feyereislova A, Chung HC, Shen L, Sawaki A, et al. Trastuzumab in combination with chemother- 
apy versus chemotherapy alone for treatment of HER2-positive advanced gastric or gastro-oesophageal junction cancer (ToGA): a phase 3, open-label, randomised controlled trial. Lancet. 2010;376:687-97.

7. Jung KW, Won YJ, Kong HJ, Oh CM, Shin A, Lee JS. Survival of Korean adult cancer patients by stage at diagnosis, 20062010: national cancer registry study. Cancer Res Treat. 2013;45: 162-71.

8. Seo SH, Kim SE, Kang YK, Ryoo BY, Ryu MH, Jeong JH, et al. Association of nutritional status-related indices and chemotherapy-induced adverse events in gastric cancer patients. BMC Cancer. 2016;16:900.

9. Lim JS, Kim SB, Bang HY, Cheon GJ, Lee JI. High prevalence of osteoporosis in patients with gastric adenocarcinoma following gastrectomy. World J Gastroenterol. 2007;13:6492-7.

10. Yu W, Park KB, Chung HY, Kwon OK, Lee SS. Chronological changes of quality of life in long-term survivors after gastrectomy for gastric cancer. Cancer Res Treat. 2016;48:1030-6.

11. Teng GG, Curtis JR, Saag KG. Mortality and osteoporotic fractures: is the link causal, and is it modifiable? Clin Exp Rheumatol. 2008;26(5 Suppl 51):S125-37.

12. Yoo SH, Lee JA, Kang SY, Kim YS, Sunwoo S, Kim BS, et al. Risk of osteoporosis after gastrectomy in long-term gastric cancer survivors. Gastric Cancer. 2018;21:720-7.

13. Oh HJ, Lim CH, Yoon BH, Yoon SB, Baeg MK, Kim WC, et al. Fracture after gastrectomy for gastric cancer: a long-term follow-up observational study. Eur J Cancer. 2017;72:28-36.

14. Kanis J, Johnell O, Gullberg B, Allander E, Elffors L, Ranstam J, et al. Risk factors for hip fracture in men from southern Europe: the MEDOS study. Mediterranean Osteoporosis Study. Osteoporos Int. 1999;9:45-54.

15. Kweon S, Kim Y, Jang MJ, Kim Y, Kim K, Choi S, et al. Data resource profile: the Korea National Health and Nutrition Examination Survey (KNHANES). Int J Epidemiol. 2014;43:6977.

16. Kanis JA on behalf of the World Health Organization Scientific Group. Assessment of osteoporosis at the primary health-care level. Technical report. Sheffield: World Health Organization Collaborating Centre for Metabolic Bone Diseases, University of Sheffield; 2007.

17. Kim KM, Choi HS, Choi MJ, Chung HY. Calcium and vitamin D supplementations: 2015 position statement of the Korean Society for Bone and Mineral Research. J Bone Metab. 2015;22: 143-9.

18. Heiskanen JT, Kroger H, Paakkonen M, Parviainen MT, Lamberg-Allardt C, Alhava E. Bone mineral metabolism after total gastrectomy. Bone. 2001;28:123-7.

19. Adachi Y, Shiota E, Matsumata T, Iso Y, Yoh R, Kitano S. Osteoporosis after gastrectomy: bone mineral density of lumbar spine assessed by dual-energy X-ray absorptiometry. Cal- cif Tissue Int. 2000;66:119-22.

20. Eom BW, Kim J, Kim DH, Kim YI, Yoon HM, Cho SJ, et al. Recovery of food intake after gastrectomy for gastric cancer: based on a large-scale gastric cancer cohort. Dig Surg. 2018;35: 220-9.

21. Baek KH, Jeon HM, Lee SS, Lim DJ, Oh KW, Lee WY, et al. Short-term changes in bone and mineral metabolism following gastrectomy in gastric cancer patients. Bone. 2008;42:61-7.

22. Recker RR. Calcium absorption and achlorhydria. N Engl J Med. 1985;313:70-3.

23. Oh MG, Han MA, Park J, Ryu SY, Choi SW. The prevalence of vitamin $\mathrm{D}$ deficiency among cancer survivors in a nationwide survey of the Korean population. PLoS One. 2015;10:e0129901.

24. Straatman J, Wiegel J, van der Wielen N, Jansma EP, Cuesta MA, van der Peet DL. Systematic review of exocrine pancreatic insufficiency after gastrectomy for cancer. Dig Surg. 2017; 34:364-70.

25. Jin J, Robinson AV, Hallowell PT, Jasper JJ, Stellato TA, Wilhem SM. Increases in parathyroid hormone (PTH) after gastric bypass surgery appear to be of a secondary nature. Surgery. 2007;142:914-20.

26. Costa AG, Bilezikian JP. Bone turnover markers in primary hyperparathyroidism. J Clin Densitom. 2013;16:22-7.

27. Stein EM, Carrelli A, Young P, Bucovsky M, Zhang C, Schrope $\mathrm{B}$, et al. Bariatric surgery results in cortical bone loss. J Clin Endocrinol Metab. 2013;98:541-9.

28. Duan Y, De Luca V, Seeman E. Parathyroid hormone deficiency and excess: similar effects on trabecular bone but differing effects on cortical bone. J Clin Endocrinol Metab. 1999; 84:718-22.

29. Cheng N, Green ME. Osteoporosis screening for men: are family physicians following the guidelines? Can Fam Physician. 2008;54:1140-1.e1-5.

30. Lee JE, Shin DW, Lee H, Son KY, Kim WJ, Suh YS, et al. Oneyear experience managing a cancer survivorship clinic using a shared-care model for gastric cancer survivors in Korea. J Korean Med Sci. 2016;31:859-65.

31. Bernstein CN, Leslie WD, Leboff MS. AGA technical review on osteoporosis in gastrointestinal diseases. Gastroenterology. 2003;124:795-841.

32. Kochersberger G, Bales C, Lobaugh B, Lyles KW. Calcium supplementation lowers serum parathyroid hormone levels in elderly subjects. J Gerontol. 1990;45:M159-62.

33. Cho S, Shin A, Song D, Park JK, Kim Y, Choi JY, et al. Validity of self-reported cancer history in the health examinees (HEXA) study: a comparison of self-report and cancer registry records. Cancer Epidemiol. 2017;50:16-21.

34. Cho LY, Kim CS, Li L, Yang JJ, Park B, Shin A, et al. Validation of self-reported cancer incidence at follow-up in a prospective cohort study. Ann Epidemiol. 2009;19:644-6. 